\title{
Circumscribed Palmar Hypokeratosis: Successful Treatment with Fluorouracil Cream
}

Michael Wilk · Bettina G. Zelger · Bernhard Zelger

To view enhanced content go to www.dermtherapy-open.com

Received: March 5, 2011 / Published online: May 4, 2011

(C) The Author(s) 2011. This article is published with open access at Springerlink.com

\section{ABSTRACT}

Introduction: The pathogenesis of circumscribed palmar hypokeratosis $(\mathrm{CPH})$ is still controversial, and to date there is no treatment of choice. Methods: We report on a patient with $\mathrm{CPH}$ responding to treatment with fluorouracil cream. Results: The clinical course and histopathological features in our observation point towards the possibility of an underlying squamous cell carcinoma in situ presenting as $\mathrm{CPH}$. Conclusion: We suggest that fluorouracil cream treatment should be considered as a therapeutic option in cases of $\mathrm{CPH}$.

Keywords: circumscribed palmar hypokeratosis; dermatologic therapy; dermatopathology; fluorouracil cream

Michael Wilk $(\bowtie)$

Dermatohistological Laboratory and Private Practice, Postfach 4145, 90021 Nuernberg, Germany.

Email: M.Wilk@t-online.de

Bettina G. Zelger

Institute of Pathology, Medical University Innsbruck, Innsbruck, Austria

Bernhard Zelger

Clinical Department of Dermatology \& Venereology,

Medical University Innsbruck, Innsbruck, Austria

\section{INTRODUCTION}

The pathogenesis of circumscribed palmar hypokeratosis (CPH) is still controversial, with several different hypotheses having been considered so far. Similarly, various therapies have been applied, and none have been established as a treatment of choice. In this paper, we report on a multifocal case of $\mathrm{CPH}$ responding to treatment with fluorouracil cream.

\section{REPORT}

A 67-year-old female presented with a 10-year history of a nonhealing circular erythematous depressed lesion on her right index finger measuring $12 \mathrm{~mm}$ in diameter. The lesion gradually healed after 9 months treatment with fluorouracil cream, applied 1-2 times daily with intermittent occlusion. There was no recurrence, with a follow-up of 4.5 years. She subsequently developed three similar lesions, two of which were on her left thenar eminence and one on her left distal medial middle finger, measuring 5,3 , and $4 \mathrm{~mm}$ in diameter, respectively. There was no preceding trauma. The lesions on the 
thenar responded to intermittently-applied fluorouracil cream within 18 months, but recurred 3 months after cessation of treatment (Figure 1). The lesion on the left middle finger did not show a significant improvement.

Histopathology of a biopsy of the left thenar obtained from the margin of the lesion (Figure 2) showed a lateral aspect of normal volar skin with orthokeratosis, descending in a frayed fashion to an area with mild parakeratosis and hypogranulosis. No significant atypia of keratocytes was noted; however, the lesional part of the epidermis stained fainter, compared with the noninvolved epidermis. There was a moderate dilatation of capillaries in the papillary dermis. Some bacteria (due to their dotted and rod-like appearance, probably corynebacteria) were seen in the frayed portion of the margin. The periodic acid-Schiff (PAS) stain did not reveal any fungi. An in-situ hybridization test for low- and high-risk papillomavirus was negative.

Figure 1. Recurrence of two lesions of circumscribed palmar hypokeratosis on the left thenar eminence.

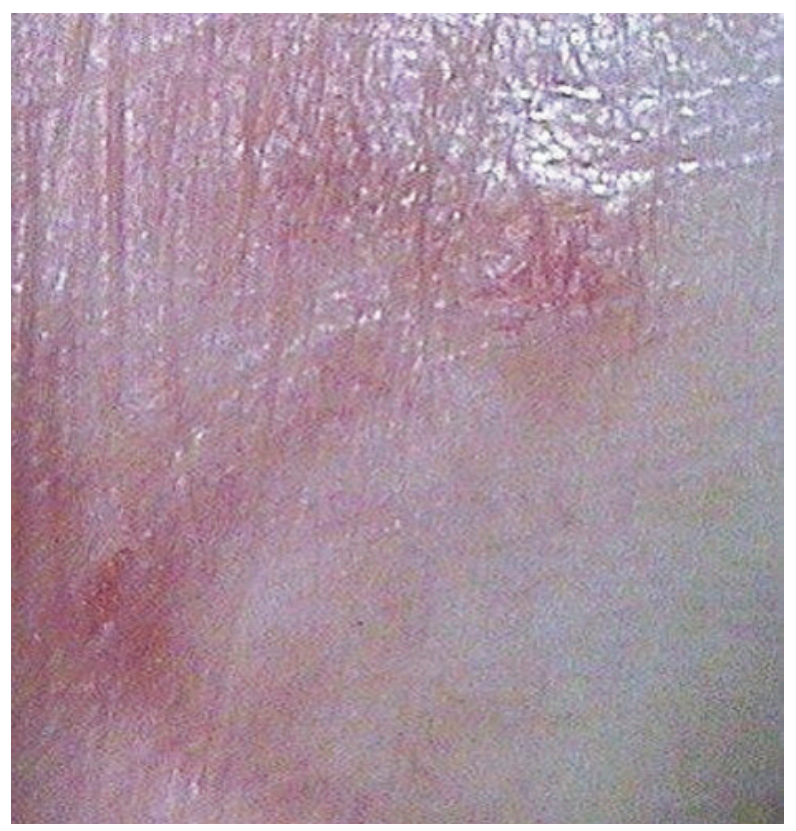

Figure 2. Biopsy from the margin of the left thenar lesion (upper lesion in Figure 1). Lateral portion of normal skin descending in a frayed fashion to an area of mild parakeratosis and hypogranulosis; moderate dilatation of superficial capillaries. Note the sharp demarcation with different quality of epidermal staining, which is fainter within the lesion. In addition, bacteria with dotted and rod-like appearance are present in the frayed portion. Hematoxylin and eosin stain (H\&E) x 100 and x 400 .

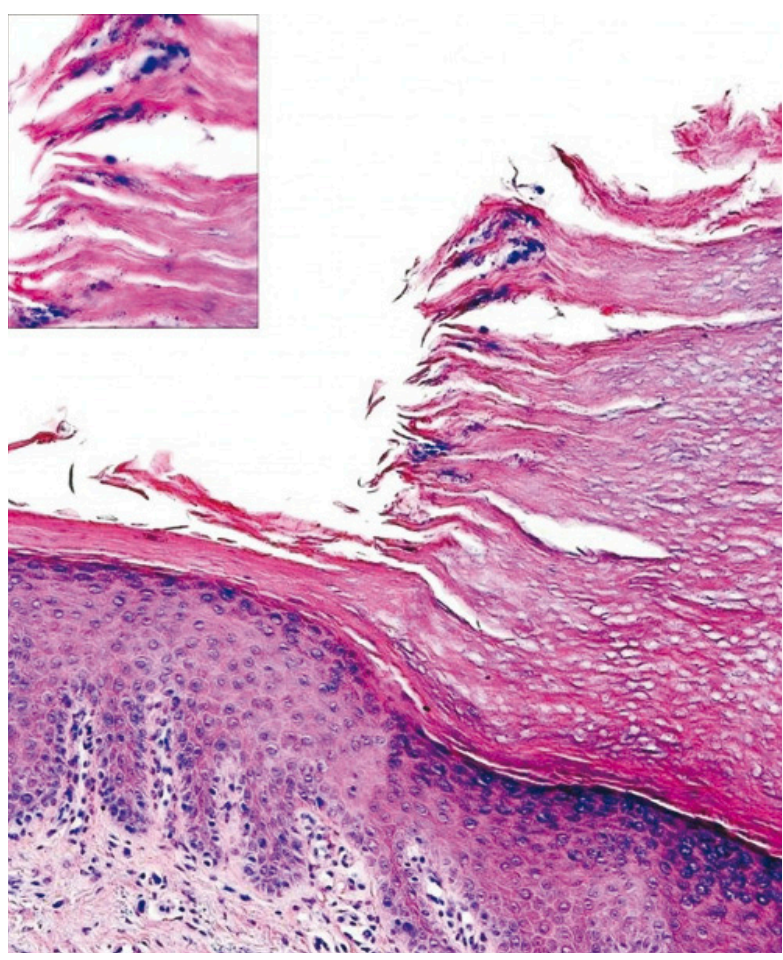

\section{DISCUSSION}

$\mathrm{CPH}$ was first described in 2002 in a series of 10 patients. ${ }^{1}$ There was a striking female predominance, and the lesions usually presented in middle-aged to elder adults as a long-standing solitary palmar area of erythematous depressed skin. Multiple lesions, ${ }^{2-4}$ as in the present observation and in plantar presentation, ${ }^{1,5,6}$ appeared to be the exception. In a few patients, repeated trauma was reported to play a role in the pathogenesis. ${ }^{3,7}$ However, most patients, including the one in the present study, cannot recall this, ${ }^{1,2,8}$ and there is no explanation as to why the majority of lesions do not tend to 
heal spontaneously. Pérez et al. ${ }^{1}$ considered that the disease might result from a clone of abnormal keratocytes, but in view of its benign behavior, favored an epidermal malformation. This theory has been challenged due to its late onset in life and occasional enlargement. ${ }^{2}$ Other hypotheses include a variant of epidermolysis bullosa, ${ }^{3}$ a defect in keratinization, ${ }^{5,9}$ which has been supported by the finding of altered keratin expression, ${ }^{6}$ and a hyperproliferative epidermal state, along with enhanced corneocyte fragility. ${ }^{4}$ Case reports detected human papillomavirus type 6 and $4,{ }^{10}$ a finding which was not present in two examined specimens of the original series, ${ }^{1}$ other studies, ${ }^{4,5,11}$ and in the present observation.

An interesting histopathological finding in our patient that has not been commented on previously was the presence of rod-like bacteria, likely representing corynebacteria. They are presumably coincidental. However, in the right clinical setting, pitted keratolysis must be considered in the differential diagnosis.

In addition, parakeratosis is a feature which has been reported in other publications as well,,$^{3,8,11,12}$ but has not been present in the cases of the original series ${ }^{1}$ and other reports, ${ }^{5,8}$ possibly pointing towards different evolutionary stages, ${ }^{8}$ malignant transformation, ${ }^{11}$ or different diseases. Parakeratosis per se is a nonspecific finding, but together with the presence of a fainter stained epidermis, hypogranulosis, and dilated vessels in the papillary dermis, might point towards an underlying squamous cell carcinoma in situ presenting as $\mathrm{CPH}$. The clinical course in the present patient supports this hypothesis. In contrast to a previous observation, ${ }^{10}$ most of the lesions responded to treatment with fluorouracil cream, with the lesions on the left thenar showing recurrence later on. Failure of the left middle fingers lesion to heal and the long period of treatment for the other lesions are possibly due to their distal location, with impaired effectiveness of fluorouracil cream during activities such as daily housework.

To date, no other treatment has been shown to be the treatment of choice in $\mathrm{CPH}$. Steroids, immunomodulators, retinoids, and hydrocolloid dressings have been applied without success. ${ }^{1,4,7,11}$ Calcipotriol led to improvement in a number of patients, ${ }^{1,9}$ while it failed to have an effect in others. ${ }^{4,8}$ Further case reports documented partial remission by photodynamic therapy, ${ }^{12}$ and complete healing with cryotherapy. ${ }^{7}$ Local excision was performed in another case and was curative with a follow-up of 6 months. ${ }^{8}$ One patient with bilateral involvement experienced spontaneous healing. ${ }^{3}$

Although one should be cautious in generalizing the outcome of a single case, from the present observation we suggest that fluorouracil cream treatment should be considered as a therapeutic option in cases of $\mathrm{CPH}$ when the histopathological features point toward the possibility of an underlying squamous cell carcinoma in situ.

\section{ACKNOWLEDGMENTS}

The authors declare that they have no conflicts of interest, and no funding or sponsorship was received in relation to this study. Michael Wilk is the guarantor for this article, and takes responsibility for the integrity of the work as a whole.

Open Access. This article is distributed under the terms of the Creative Commons Attribution Noncommercial License which permits any noncommercial use, distribution, and reproduction in any medium, provided the original author(s) and source are credited. 


\section{REFERENCES}

1. Pérez A, Rütten A, Gold R, et al. Circumscribed palmar or plantar hypokeratosis: a distinctive epidermal malformation of the palms and soles. J Am Acad Dermatol. 2002;47:21-27.

2. Obermoser G, Zelger B. "Multifocal" circumscribed palmar hypokeratosis: malformation or not? J Am Acad Dermatol. 2003;49:1197-1198.

3. Resnik K, DiLeonardo M. Circumscribed palmar hypokeratosis. New observations. Am J Dermatopathol. 2006;28:112-116.

4. Ishiko A, Dekio I, Fujimoto A, et al. Abnormal keratin expression in circumscribed palmar hypokeratosis. J Am Acad Dermatol. 2007;57:285291.

5. Barry CI, Glusac EJ, Kashgarian M, Gwin K, McNiff JM. Circumscribed palmar hypokeratosis: two cases and a review of the literature. J Cutan Pathol. 2008;35:484-487.

6. Tanioka M, Miyagawa-Hayashino A, Manabe T, Toichi E, Miyachi Y, Takahashi K. Circumscribed palmo-plantar hypokeratosis: a disease with two subtypes. J Invest Dermatol. 2009;129:1045-1047.
7. Boffa MJ, Degaetano JS. Circumscribed palmar hypokeratosis: successful treatment with cryotherapy. J Eur Acad Dermatol Venereol. 2007;21:420-421.

8. Mensing $\mathrm{CH}$, Schleusner VH, Sander CA, Mensing $\mathrm{H}$. Circumscribed palmar or plantar hypokeratosis. Two cases of a recently described entity of unknown origin. Am J Dermatopathol. 2005;27:247-249.

9. Urbina F, Misad C, Gonzalez S. Circumscribed palmar hypokeratosis: clinical evolution and ultrastructural study after prolonged treatment with topical calcipotriol. J Eur Acad Dermatol Venereol. 2005;19:491-494.

10. Berk DR, Böer A, Bauschard FD, Hurt MA, Santa-Cruz DJ, Eisen AZ. Circumscribed acral hypokeratosis. J Am Acad Dermatol. 2007;57:292296.

11. Kanitakis J, Lora V, Balme B, Roby J. Premalignant circumscribed palmar hypokeratosis: A new form of circumscribed palmar hypokeratosis? Case report and literature review. Dermatology. 2010;220:143146.

12. Benoit S, Seitz CS, Hamm H, Vetter-Kauczok CS, Bröcker E-B. Circumscribed palmar hypokeratosis: partial remission by photodynamic therapy. $\mathrm{Br} \mathrm{J}$ Dermatol. 2007;157:804-806. 